\title{
Low-Sampling Rate UWB Channel Characterization and Synchronization
}

\author{
Irena Maravić, Julius Kusuma and Martin Vetterli
}

\begin{abstract}
We consider the problem of low-sampling rate high-resolution channel estimation and timing for digital ultra-wideband (UWB) receivers. We extend some of our recent results in sampling of certain classes of parametric nonbandlimited signals and develop a frequency domain method for channel estimation and synchronization in ultra-wideband systems, which uses sub-Nyquist uniform sampling and wellstudied computational procedures. In particular, the proposed method can be used for identification of more realistic channel models, where different propagation paths undergo different frequency-selective fading. Moreover, we show that it is possible to obtain high-resolution estimates of all relevant channel parameters by sampling a received signal below the traditional Nyquist rate. Our approach leads to faster acquisition compared to current digital solutions, allows for slower A/D converters, and potentially reduces power consumption of digital UWB receivers significantly.
\end{abstract}

Index Terms: UWB, multipath channel, channel modeling, channel estimation, sampling, annihilating filters, timing.

\section{INTRODUCTION}

Ultra-wideband (UWB) technology has recently received much attention for many short-range applications, such as accurate ranging and positioning as well as multipath fading mitigation in indoor wireless networks [1] [2] [3] [4] [5]. UWB signals are generated by driving an antenna with very short electrical pulses, typically on the order of a nanosecond, thus spreading the signal energy from near DC to a few gigahertz. Although the possibility of using extremely short pulses for certain applications (such as ranging or imaging) has been investigated for at least two decades, there still remains a lot to be done for this technology to become pervasive. Some of the important issues include low-cost and low-power designs and novel signal processing techniques that allow for efficient digital implementation.

The wideband nature of UWB brings new research challenges both in the analysis and practice of reliable systems. The first challenge is rapid synchronization and acquisition for UWB systems. There is a vast literature that has appeared recently [1] [2] [6] [7] [8] [9], addressing both algorithmic and implementation issues of several synchroniza-

\footnotetext{
This work was partially presented at the IEEE International Conference on Communications 2003, May 2003, Alaska, and the International Workshop on Ultra-Wideband Systems (IWUWB) 2003, June 2003, Finland. Irena Maravić is with LCAV, Swiss Federal Institute of Technology, Lausanne, Switzerland. e-mail: irena.maravic@epfl.ch. Julius Kusuma is with the Lab. for Information and Decision Systems, MIT. e-mail: kusuma@mit.edu. Martin Vetterli is with LCAV, Swiss Federal Institute of Technology, Lausanne, Switzerland, and the Dept. of EECS, U.C. Berkeley. e-mail: martin.vetterli@epfl.ch
}

tion techniques, with a clear trend to minimize needed analog components and perform as much processing digitally as possible [6] [9]. Digital implementation has well-known advantages, including cheaper technology, full integration, robustness etc. However, given the extreme bandwidths involved, it still represents a design challenge. While highperformance schemes have already been proposed for ana$\log$ systems [7], their application to digital-oriented solutions is still not feasible due to prohibitively high computational requirements. Furthermore, implementation of such techniques in digital systems would require very fast and expensive A/D converters (operating in the gigahertz range) and therefore will result in high power consumption. Finally, they are mostly based on exhaustive search and are inherently time-consuming.

The second challenge arises from the fact that UWB propagation models in multipath environments are more complex than existing narrowband models and do not allow for direct extension of narrowband techniques. The finer time resolution of UWB means that different multipath components arriving at the receiver at different delays and at different angles create a dynamic and extended channel impulse [3] [4] [5]. To more accurately characterize ultrawideband channels, a new model was proposed in which different incoming paths undergo different frequency-selective attenuations [1]. In their paper, Cramer, Scholtz and Win proposed to spatially separate the multipath components, which is then followed by identifying the different frequency-selective fading of the individual components. However, this requires additional hardware, in form of an antenna array. They used the CLEAN algorithm for the processing of the signal, which was first developed for space telemetry applications.

Starting with a simplified channel model, we develop a method which inspires a new approach to channel estimation and synchronization in wideband systems. This method yields very precise estimates of channel parameters, and uses sub-Nyquist uniform sampling and welldeveloped algorithmic solutions. Specifically, we extend some of our recent sampling results for certain classes of non-bandlimited signals [10] to the problem of channel estimation in ultra-wideband systems [11] [12] [13] [14] [15], where unknown channel parameters are estimated from a low-dimensional signal subspace. Our approach leads to reduced computational requirements and faster acquisition compared to other proposed digital techniques [6] [8] [9], thus allowing for a practical hardware implementation and lower power consumption. We will then extend this framework to a more realistic channel model given in [1]. We 
show that our approach can be used to characterize a more general channel model without requiring additional hardware support. Our method is particularly suitable in applications such as estimation of wideband channels, precise position location or ranging. It can be used in other wideband systems as well, primarily for timing synchronization and localization purposes.

The outline of the paper is as follows. In Section II, we introduce an UWB channel model and discuss the advantages of the frequency-domain approach, as well as prior work in spectral estimation. In Section III, we present an algorithm based on annihilating filters, which allows for joint estimation of pulse shapes and time delays along different propagation paths. We also discuss a possible modification of the algorithm which leads to improved performance in the presence of noise. Analysis of noise sensitivity and computational complexity are discussed in Section IV. In Section V, we present some simulation results that indicate the effectiveness of our approach, showing performances that exceed those of conventional methods. Finally, in Section VI, we conclude with a brief summary of our work.

\section{Problem Statement}

Many communication systems require the receiver and/or the transmitter to know the channel impulse response. Other applications, most notably GPS and UWBbased ranging systems, require very accurate timing estimation. All of these systems typically use very high sampling frequencies, or involve complex hardware systems to enable highly-accurate timing estimation.

Consider the following simple example. Let the signal $x(t)$ be a sum of delta functions with unknown delays $\left\{t_{l}\right\}_{l=0}^{L-1}$ and weighting coefficients $\left\{a_{l}\right\}_{l=0}^{L-1}$,

$$
x(t)=\sum_{l=0}^{L-1} a_{l} \delta\left(t-t_{l}\right) .
$$

Note that the Fourier transform of this signal is given by:

$$
X(\omega)=\sum_{l=0}^{L-1} a_{l} e^{j \omega t_{l}} .
$$

By considering the frequency domain representation of the signal, we can convert the problem of estimating the unknown parameters $\left\{t_{l}\right\}_{l=0}^{L-1}$ and $\left\{a_{l}\right\}_{l=0}^{L-1}$ into the classic spectral estimation problem, that is, estimating complex frequencies and weighting coefficients of superimposed exponentials.

The problem of high-resolution spectral estimation is well-studied: there exists a rich body of literature on both the theoretical limits and efficient algorithms for reliable estimation [16] [17] [18] [19]. There is a particularly attractive class of subspace or SVD-based algorithms, called super-resolution methods, which can resolve closely spaced sinusoids from a short record of noise-corrupted data. In [19], a state space method is proposed, which provides an elegant and numerically robust tool for parameter estimation using a model-based approach. The ESPRIT algorithm is developed in [18], which can be viewed as a generalization of the state space method applicable to general antenna arrays. In [17], several SVD-based techniques for estimating generalized eigenvalues of matrix pencils are addressed, such as Direct matrix pencil algorithm, ProESPRIT and its improved version TLS-ESPRIT.

The parameter estimation problem also arises in the context of channel estimation in other wideband systems, such as DS-CDMA [20] [21]. Even though methods developed for DS-CDMA can be adapted to UWB systems, much higher sampling rates in the latter as well as certain bandwidth-dependent effects make the modeling and estimation of UWB channels a more difficult problem and therefore require a different solution.

\section{A. UWB Channel Estimation}

A number of propagation studies for ultra-wideband signals have been done, which take into account temporal properties of a channel or characterize a spatio-temporal channel response [1] [22]. A typical model for the impulse response of a multipath fading channel is given by

$$
h(t)=\sum_{l=1}^{L} a_{l} \delta\left(t-t_{l}\right)
$$

where $t_{l}$ denotes a signal delay along the $l$-th path while $a_{l}$ is a complex propagation coefficient, which includes a channel attenuation and a phase offset along the $l$-th path. Although this model does not adequately reflect specific frequency-dependent effects, it is commonly used for diversity reception schemes in conventional wideband receivers (e.g. RAKE receivers) [1] [23]. Equation (3) means that a received signal $y(t)$ consists of a weighted sum of attenuated and delayed replicas of a transmitted signal $s(t)$, i.e.,

$$
y(t)=\sum_{l=1}^{L} a_{l} s\left(t-t_{l}\right)+z(t)
$$

where $z(t)$ denotes receiver noise. Clearly, the problem of estimating unknown channel parameters $a_{l}$ and $t_{l}$ is a special case of above mentioned spectral estimation problems.

However, different key parameters affect the behavior of wideband channels as the bandwidth is increased. The finer time resolution of UWB means that different multipath components arriving at the receiver at different delays and at different angles create a dynamic and extended channel impulse [3] [4] [5]. To more accurately characterize ultra-wideband channels, a new model was proposed in [1], in which different incoming paths undergo different frequency-selective attenuations. This model can be written as:

$$
h(t)=\sum_{l=1}^{L} a_{l} p_{l}\left(t-t_{l}\right)
$$

Thus, a received signal is made up of pulses having different 
pulse shapes,

$$
y(t)=\sum_{l=1}^{L} a_{l} s_{l}\left(t-t_{l}\right)+z(t)
$$

where $s_{l}(t)$ are different pulse shapes that correspond to different propagation paths. Let $Y(\omega)$ denote the Fourier transform of the received signal

$$
Y(\omega)=\sum_{l=1}^{L} a_{l} S_{l}(\omega) e^{-j \omega t_{l}}+Z(\omega)
$$

where $S_{l}(\omega)$ and $Z(\omega)$ are Fourier transforms of $s_{l}(t)$ and $z(t)$ respectively. Clearly, in order to completely characterize the channel, we need to estimate the $a_{l}$ 's and $t_{l}$ 's as well as $S_{l}(\omega)$.

In previous work [1], Cramer, Scholtz and Win used an antenna array to achieve spatio-temporal separation of the received signal components. We are interested in low-complexity, possibly even single-antenna applications, where the receiver can resolve the different multipath components without resorting to spatial separation. In the following section, we introduce a frequency-domain method which takes advantage of the fact that the multipath components will be subjected to independent delays at the reception.

\section{Channel Estimation Method}

\section{A. Theory}

Let the ideal bandpass filter be given by:

$$
H_{b}=\operatorname{rect}\left(\omega_{L}, \omega_{U}\right)=1_{\left[\omega_{L}, \omega_{U}\right]}
$$

where $\omega_{L}$ and $\omega_{U}$ are the cutoff frequencies, while the central frequency is $\omega_{c}=\frac{\omega_{L}+\omega_{U}}{2}$, and let $h_{b}(t)$ be the timedomain representation of this ideal bandpass filter.

Suppose that the received signal $y(t)$ is filtered with an ideal bandpass filter with cutoff frequencies $\omega_{L}$ and $\omega_{U}$ and sampled uniformly at a rate $R_{s} \geq \frac{\omega_{U}-\omega_{L}}{2 \pi}$. Assume next that $N=2 M+1$ uniformly spaced frequency samples of $Y(\omega)$ are available, that is,

$$
Y[n]=Y\left(\omega_{c}+n \omega_{0}\right), \quad \omega_{0}=\frac{\omega_{U}-\omega_{L}}{2 M}, \quad n=-M, \ldots, M .
$$

If we denote by $\omega_{n}=\omega_{c}+n \omega_{0}$, the samples $Y[n]$ can be expressed as

$$
Y[n]=\sum_{l=1}^{L} a_{l} S_{l}[n] e^{-j \omega_{n} t_{l}}+Z[n]
$$

where $S_{l}[n]$ are the samples of the Fourier transform $S\left(\omega_{n}\right)$ of the received UWB pulses. Note that in practice, the discrete Fourier transform (DFT) will be used to determine $Y[n]$ and $S_{l}[n]$, therefore, in general, (9) and (10) will not hold exactly. However, these equations are asymptotically accurate, provided that the sampling period is sufficiently small to avoid aliasing at the output of the bandpass filter since the error introduced by a finite length DFT is on the order of $\mathcal{O}\left(N^{-1}\right)$. Note that this does not imply that the sampling rate has to be above the Nyquist rate of the original signal, but rather the Nyquist rate which is dictated by the bandpass filter.

In general, the problem of estimating all the unknown parameters requires a non-linear estimation procedure. One possible way to convert the nonlinear estimation problem into the simpler problem of estimating the parameters of a linear model is to approximate the coefficients $S_{l}[n]$ with polynomials of degree $d \leq R-1$, that is,

$$
S_{l}[n]=\sum_{r=0}^{R-1} s_{l, r} n^{r} .
$$

Equation (7) now becomes

$$
Y[n]=\sum_{l=1}^{L} a_{l} \sum_{r=0}^{R-1} s_{l, r} n^{r} e^{-j \omega_{n} t_{l}}+Z[n]
$$

By denoting $c_{l, r}=a_{l} s_{l, r}$, we obtain

$$
Y[n]=\sum_{l=1}^{L} \sum_{r=0}^{R-1} c_{l, r} n^{r} e^{-j \omega_{n} t_{l}}+Z[n]
$$

Note that the coefficients $Y[n]$ are again given by a sum of weighted exponentials, yet the weights depend on $n$ (through the term $n^{r}$ ). In the following, we will present a method based on annihilating filters [10] [15] [16], which allows for joint estimation of all the unknown parameters $\left(c_{l, r}\right.$ and $\left.t_{l}\right)$ from a set of at least $2 R L+1$ coefficients $Y[n]$.

The main idea behind this approach is to find the socalled annihilating filter $H(z)=\sum_{k=0}^{N} H[k] z^{-k}$ that satisfies

$$
(H * Y)[n]=0, \quad \forall n \in \mathbb{Z} .
$$

It can be shown that such a filter has multiple roots at $z_{l}=e^{-j \omega_{0} t_{l}}[10]$, that is,

$$
H(z)=\prod_{l=1}^{L}\left(1-e^{-j \omega_{0} t_{l}} z^{-1}\right)^{R}=\sum_{k=0}^{R L} H[k] z^{-k}
$$

where $\omega_{0}=\frac{\omega_{U}-\omega_{L}}{2 M}(9)$. Therefore, the information about the time delays $t_{l}$ can be extracted from the roots of the filter $H(z)$. The corresponding pulse shapes can be then estimated by solving for the coefficients $c_{l, r}$ in (13). In the following, we give an outline of the algorithm, while a more detailed analysis of the annihilating filters can be found in [10] [15] [16].

\section{B. Algorithm outline}

1. Compute the spectral coefficients $Y[n]$ from the set of samples

$$
y_{n}=<h_{b}\left(t-n T_{s}\right), y(t)>, \quad n=1, \ldots, N
$$

where $T_{s}=1 / R_{s}$ and $N \geq 2 L$. 
2. Find the coefficients $H[k]$ of the annihilating filter

$$
H(z)=\prod_{l=1}^{L}\left(1-e^{-j \omega_{0} t_{l}} z^{-1}\right)^{R}=\sum_{k=0}^{R} H[k] z^{-k}
$$

which satisfies

$$
H[n] * Y[n]=\sum_{k=0}^{R L} H[k] Y[n-k]=0, \quad \forall n \in \mathbb{Z}
$$

In matrix form, the system (18) is equivalent to

$$
\left(\begin{array}{cccc}
\vdots & \vdots & \ldots & \vdots \\
Y[1] & Y[0] & \cdots & Y[-(R L-1)] \\
Y[2] & Y[1] & \ldots & Y[-(R L-2)] \\
\vdots & \vdots & \ddots & \\
Y[R L] & Y[R L-1] & \cdots & Y[0] \\
\vdots & \vdots & &
\end{array}\right) \cdot\left(\begin{array}{c}
H[0] \\
H[1] \\
\vdots \\
H[R L]
\end{array}\right)=\mathbf{0}
$$

Since there are $R L+1$ unknown filter coefficients, we need at least $R L+1$ equations, therefore, the number of DFT coefficients we have to compute is at least $2 R L+1$.

3 . Find the values of $t_{l}$ by finding the roots of $H(z)$. At this point, it is worth noting that while this is true in the noiseless case, in the presence of noise, it is more desirable to estimate the time delays from $L$ roots of $H(z)$ which are closest to the unit circle.

4. Solve for the coefficients $c_{l, r}$ by solving the system of linear equations in (13),

$$
Y[n]=\sum_{l=1}^{L} \sum_{r=0}^{R-1} c_{l, r} n^{r} e^{-j \omega_{n} t_{l}}
$$

A block diagram of the system implementing the above algorithm is shown in Figure 1.

\section{Algorithm in the Presence of Noise}

In the theoretical case of noiseless data, any subspace of sufficient dimension ${ }^{1}$, can be used to estimate all the relevant parameters. In practice, noise will be present, and this can be dealt with by oversampling and using standard techniques in noisy spectral estimation, such as the singular value decomposition (SVD) [16]. Besides, in the presence of noise, it is desirable to estimate the channel from a frequency band where a signal-to-noise ratio (SNR) is highest. This brings us to a more practical version of the above algorithm, which yields robust estimates by properly exploiting the properties of the signal subspace [15] [17] [19].

Consider again the system of equations (19). By setting $H[0]=1$, the system can be rewritten as $\mathbf{Y} \cdot \mathbf{h}=-\mathbf{y}$, where

\footnotetext{
${ }^{1}$ By "sufficient dimension", we assume the dimension of the subspace (per unit of time) that allows us to represent the space of signals of interest.
}

the matrices $\mathbf{Y}, \mathbf{h}$ and $\mathbf{y}$ are defined as

$$
\mathbf{Y}=\left(\begin{array}{cccc}
\vdots & \vdots & \cdots & \vdots \\
Y[0] & Y[-1] & \cdots & Y[-(R L-1)] \\
Y[1] & Y[0] & \cdots & Y[-(R L-2)] \\
\vdots & \vdots & \ddots & \\
Y[R L-1] & Y[R L-2] & \cdots & Y[0] \\
\vdots & \vdots & &
\end{array}\right)
$$

The key is to observe two properties of the matrix $\mathbf{Y}$. The first one is that in the case of a channel with $L$ propagation paths, $\mathbf{Y}$ can be approximated with a rank- $L$ matrix [15] by computing its singular value decomposition, that is, $\mathbf{Y}=\mathbf{U}_{\mathbf{s}} \boldsymbol{\Lambda}_{\mathbf{s}} \mathbf{V}_{\mathbf{s}}{ }^{H}+\mathbf{U}_{\mathbf{n}} \boldsymbol{\Lambda}_{\mathbf{n}} \mathbf{V}_{\mathbf{n}}{ }^{H}$, where $\mathbf{U}_{\mathbf{s}}$ and $\mathbf{V}_{\mathbf{s}}$ contain $L$ principal left and right singular vectors of $\mathbf{Y}$. The second property is that both $\mathbf{U}_{\mathbf{s}}$ and $\mathbf{V}_{\mathbf{s}}$ satisfy the so-called shift-invariant subspace property [19],

$$
{\overline{\mathbf{U}_{\mathbf{s}}}}^{p}={\underline{\mathbf{U}_{\mathbf{s}}}}_{p} \cdot \boldsymbol{\Phi} \text { and }{\overline{\mathbf{V}_{\mathbf{s}}}}^{p}=\underline{\mathbf{V}}_{\mathbf{s}_{p}} \cdot \boldsymbol{\Phi}^{H}
$$

where $\boldsymbol{\Phi}$ is a diagonal matrix having elements $e^{j \omega_{0} p t_{l}}=$ $e^{j \omega_{0} \hat{t}_{l}}$ along the main diagonal, while $\overline{(\cdot)}^{p}$ and $\underline{(\cdot)}_{p}$ denote the operations of omitting the first $p$ rows and the last $p$ rows of $(\cdot)$ respectively. Therefore, the time delays $\left\{t_{l}\right\}_{l=1}^{L}$ can be uniquely determined from the eigenvalues $\lambda_{l}$ of the operator that maps $\underline{\mathbf{U}_{\mathbf{s}}}$ p onto ${\overline{\mathbf{U}_{\mathbf{s}}}}^{p}$ (or alternatively, $\underline{\mathbf{V}_{\mathbf{s}}} p$ onto ${\overline{\mathbf{V}_{\mathbf{s}}}}^{p}$ ),

$$
t_{l}=\frac{\hat{t_{l}}}{p}=\frac{N \angle \lambda_{l}}{2 \pi p}
$$

The advantage of using values of $p$ larger than $p=1$ is that the separation between the estimated time delays $\hat{t}_{l}$ is effectively increased $p$ times. This is of particular interest in the case of estimating closely spaced dominant components in low signal-to-noise-ratio (SNR) regimes (e.g. $\mathrm{SNR}<-5 \mathrm{~dB})$ when such an approach can improve the resolution performances significantly [15]. Once the time delays have been estimated, the coefficients $c_{l, r}$ are then found by solving the system (20).

Note that the information about the signal poles, and thus the time delays, can be directly extracted from the matrix $\mathbf{Y}$. That is, the above presented approach avoids the root finding part and relies only on a right deployment of matrix manipulations.

\section{Performance Evaluation}

\section{A. Analysis of Noise Sensitivity}

The statistical properties of the estimates obtained using high-resolution methods have been studied extensively, primarily in the context of estimating the frequencies of superimposed complex sinusoids from noisy measurements [16] [19]. The exact expressions for the mean-square-error (MSE) of the frequency estimates are quite complex [17], and in general do not allow for an explicit dependence of 


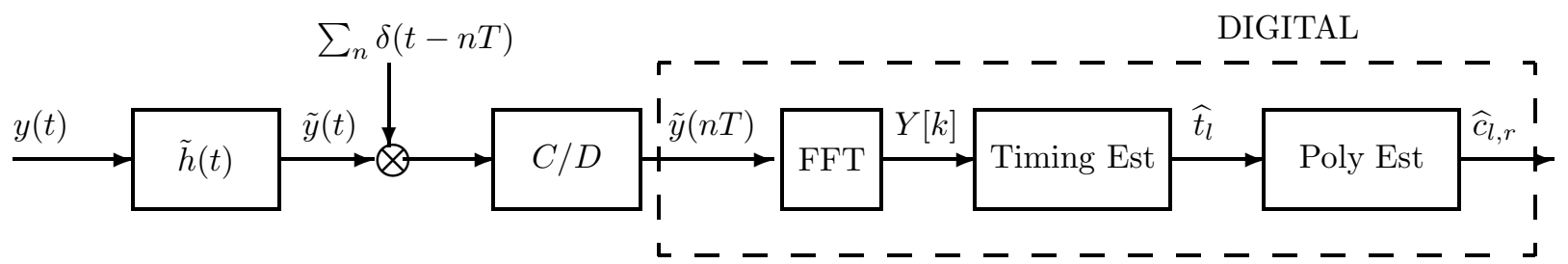

Fig. 1. Receiver block diagram

the MSE on the parameters of interest, such as the polynomial degree $R$ or the number of distinct frequencies $L$. Therefore, we give a simplified expression for the MSE in the case of a single exponential, which corresponds to the estimate of the time delay $t_{1}$ of the dominant path. Assuming that the signal and noise are uncorrelated, the MSE of the annihilating filter method can be expressed as [17],

$E\left\{\Delta t_{1}^{2}\right\} \approx \begin{cases}\frac{1}{\omega_{0}^{2}} \frac{2(2 R+1)}{3(N-R)^{2} R(R+1)} \frac{1}{\mathrm{SNR}}, & \text { for } R \leq N / 2 \\ \frac{1}{\omega_{0}^{2}} \frac{2\left(-(N-R)^{2}+3 R^{2}+3 R+1\right)}{3(N-R) R^{2}(R+1)^{2}} \frac{1}{\mathrm{SNR}}, & \text { for } R>N / 2\end{cases}$

where SNR is the signal-to-noise ratio at the output of the anti-aliasing filter at the receiver. Therefore, a choice of the polynomial degree directly affects the estimation performance. In general, a good choice for $R$ is between $N / 10$ and $9 N / 10$, while the minimum MSE is achieved for $R=N / 3$ or $R=2 N / 3$, leading to

$$
E\left\{\Delta t_{1}^{2}\right\} \approx \frac{1}{\omega_{0}^{2}} \frac{9}{N^{3}} \frac{1}{\mathrm{SNR}}
$$

This is very close to the Cramer-Rao bound (CRB) [24], which represents the lowest achievable MSE by any unbiased estimator, and is given by

$$
\mathrm{CRB}=\frac{1}{\omega_{0}^{2}} \frac{6}{N^{3}} \frac{1}{\mathrm{SNR}}
$$

We should note that the expressions for performance bounds (27) and (28) are obtained using the first order perturbation analysis and are generally valid only for medium to high signal-to-noise ratios [19]. However, these expressions provide a good basis for evaluating the performance at different sampling rates. Namely, the dependence of the root-mean-square-error (RMSE) on the number of samples $N$ is on the order of $\mathcal{O}\left(1 / N^{3 / 2}\right)$. Therefore, by decreasing the sampling rate $K$ times, RMSE increases roughly by a factor of $K^{3 / 2}$, provided that the SNR does not change. In Section V, we will show that even for very low values of SNR and sub-Nyquist sampling rates, our method gives much better performances compared to the matched-filter approach.

\section{B. Computational Complexity}

A major computational requirement of the developed algorithm is associated with the singular-valuedecomposition step in (19), which is an iterative algorithm with the computational order of $\mathcal{O}\left(N^{3}\right)$ per iteration where
$N$ denotes the total number of samples used for estimation. In [14] [15], we suggested alternative methods of lower computational requirements, which avoid explicit computation of the SVD and rely only on simple matrix multiplications. Among them are the Power method, which can be used to estimate only $L_{d}=1$ one dominant component, and the method of Orthogonal iteration, which can estimate $L_{d}>1$ paths. The computational complexity of such methods is on the order of $\mathcal{O}\left(L_{d} N^{2}\right)$, and they generally converge in less than 10 iterations. In contrast, matched-filter techniques [6] require $\mathcal{O}\left(N_{n}^{2}\right)$ operations, where $N_{n}$ denotes the number of samples taken at the Nyquist rate. Furthermore, although the matched-filter approach can be used for synchronization purposes, its time resolution is limited by the sampling rate.

\section{Simulation Results}

In this section, we show some simulation results that illustrate the performances of our algorithm. All results are based on averages of over 500 trials, each with a different realization of additive white Gaussian noise.

\section{A. UWB Timing Performance}

We first consider the case of the channel model given by (3), assuming $L=70$ propagation paths with eight dominant paths (containing $85 \%$ of the total power), as illustrated in Figure 2(a). The transmitted UWB pulse is a first-derivative Gaussian impulse with the duration of (approximately) $T_{p}=6$ samples $^{2}$, while the transmitted signal is modulated with a PN sequence of length 127 . The time delay between the transmitted pulses is 120 samples, while the average time delay between the received dominant components is 8 samples. In Figure 2(b), we show root mean square errors (RMSE) of time delay estimation for the dominant components vs. SNR (defined here as the ratio between the energy of the received sequence and a power spectral density of noise). Since we are considering the case of estimating closely spaced components for a wide range of SNR's, we used the approach presented in Section III-C, where the parameter $p$ is chosen to be $p=30$. The error is plotted for different values of the sampling rate. The results indicate that the method yields highly accurate estimates (that is, within a fraction of the pulse duration) for a wide range of SNR's, and this with sub-Nyquist sampling rates. For example, with the sam-

\footnotetext{
${ }^{2}$ Time is expressed in terms of number of samples, where one sample corresponds to the period of Nyquist-rate sampling.
} 


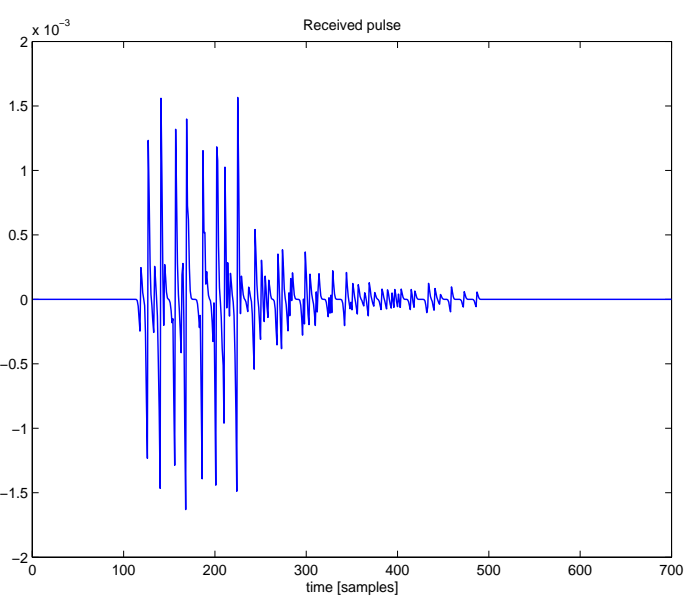

(a)

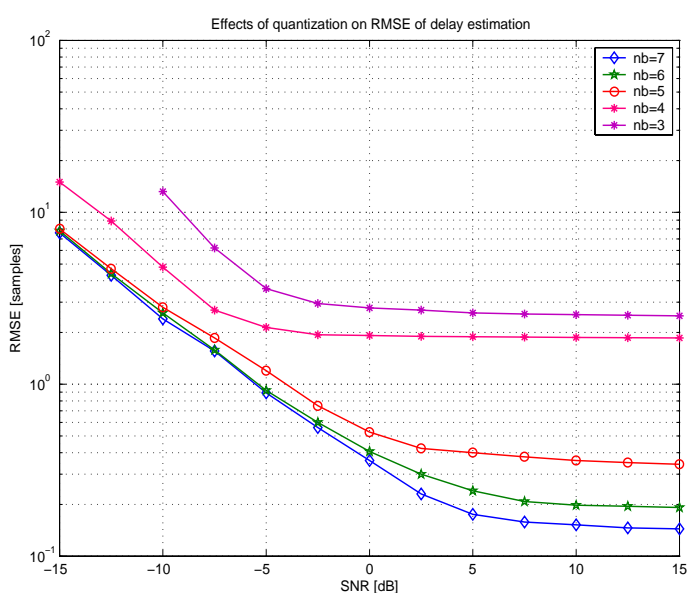

(c)

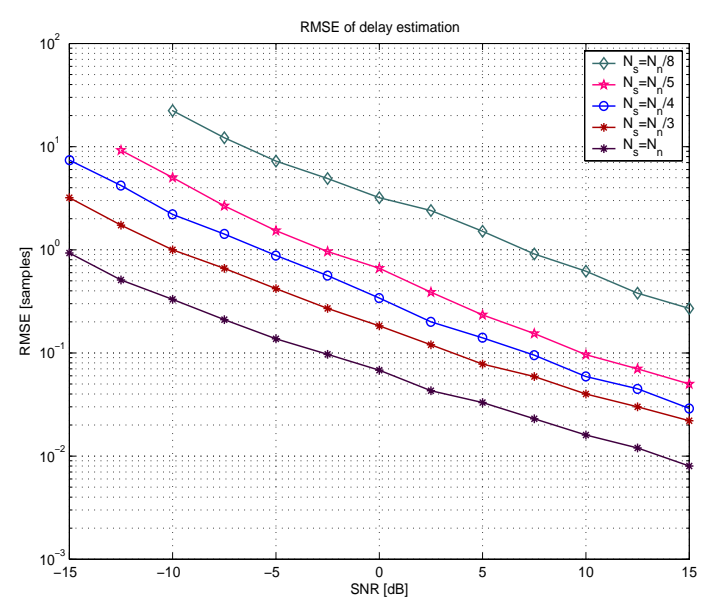

(b)

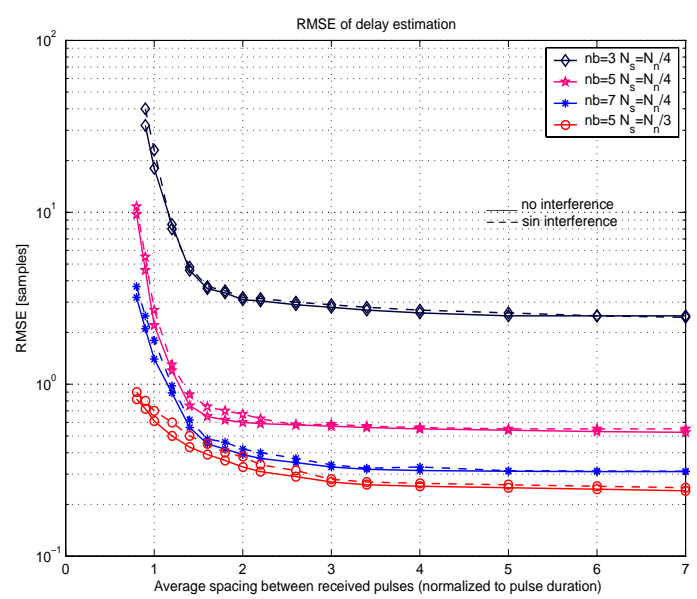

(d)

Fig. 2. Timing recovery in UWB systems (a) Received UWB signal made up of 70 pulses, with 8 components being dominant (containing approximately 85\% of the total power). The transmitted UWB pulse is an ideal first-derivative Gaussian impulse. (b) Root-mean square error (RMSE) of delay estimation (in terms of number of samples) of the dominant components vs. SNR. The error is plotted for different values of the sampling rate $N_{s}$, where $N_{n}$ denotes the Nyquist rate. (c) Effects of quantization on the RMSE of delay estimation for 3-7 bit receiver architectures. The sampling rate is one fourth the Nyquist rate ( $\left.N_{s}=N_{n} / 4\right)$. (d) RMSE of delay estimation of the dominant components vs. average spacing between the components (normalized to the pulse duration), for different number of bits used for quantization. We assumed that $S N R=-5 d B$. Dashed lines correspond to the RMSE in the presence of a strong sinusoidal interference $(S I R=-20 d B)$.

pling rate of one fourth the Nyquist rate $\left(N_{s}=N_{n} / 4\right)$ and $\mathrm{SNR}=-5 \mathrm{~dB}$, the time delay of the dominant components can be estimated with an RMSE of approximately 1 sample.

We next analyze the effects of quantization on the estimation performance. In particular, we consider 3-7 bit architectures and for each case, we plot the RMSE versus received SNR. The sampling rate is assumed to be one fourth the Nyquist rate $\left(N_{s}=N_{n} / 4\right)$. Clearly, as the number of bits increases, the overall performance improves. Generally, the 5-bit architecture already yields a very good performance. In the case when the number of bits is very low (e.g. 3 bits), quantization noise becomes dominant and determines the overall numerical performance, and this for all considered values of SNR.

In Figure 2(d), we show the RMSE of time delay estimation along the dominant paths versus the average spacing between the dominant components. The RMSE is plotted for different values of the number of quantization bits in the case when $\mathrm{SNR}=-5 \mathrm{~dB}$ and $N_{s}=N_{n} / 4$. As expected, the performance of delay estimation improves as the spacing between components is increased. Note that estimates of closely spaced paths are more sensitive to quantization [25], and increasing the sampling rate gives better performance. For example, when the sampling rate is one third the Nyquist rate, it is possible to estimate the components with an RMSE of less than 1 sample, even in the case when the average spacing between the components is only a fraction of the pulse duration.

In the same figure, we show the performance of the method in the presence of a strong sinusoidal interference. Although modulating the transmitted signal with a PN sequence is generally sufficient to suppress the interference, in the case when a signal-to-interference ratio (SIR) is very low, the processing gain may not be high enough to ensure the desirable performance. Since we solve the estimation problem in the frequency domain, we can take advantage of the fact that the sinusoidal signal is a Dirac ( $\delta$ impulse) 


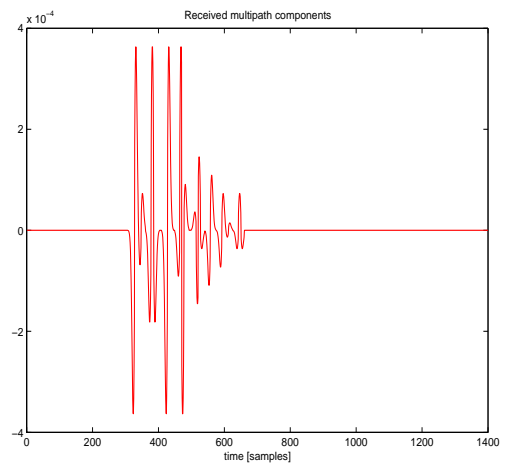

(a)

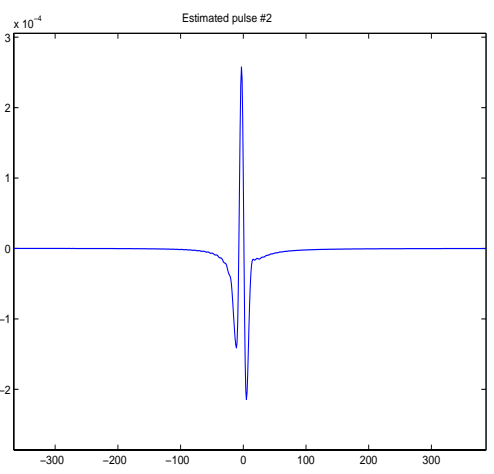

(d)

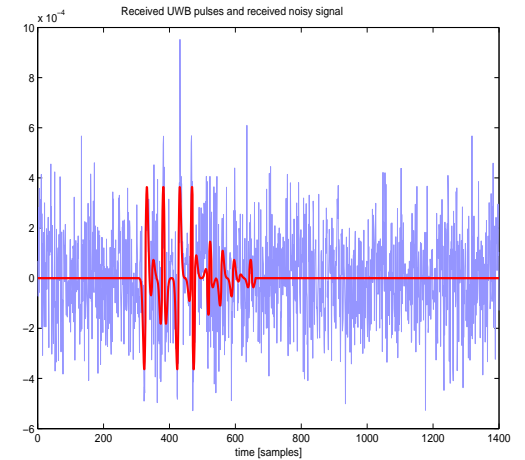

(b)

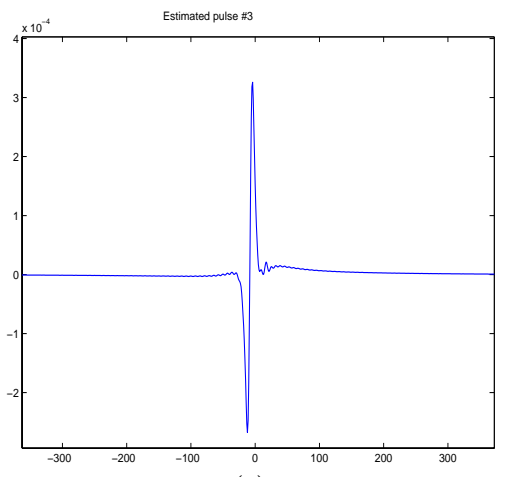

(e)

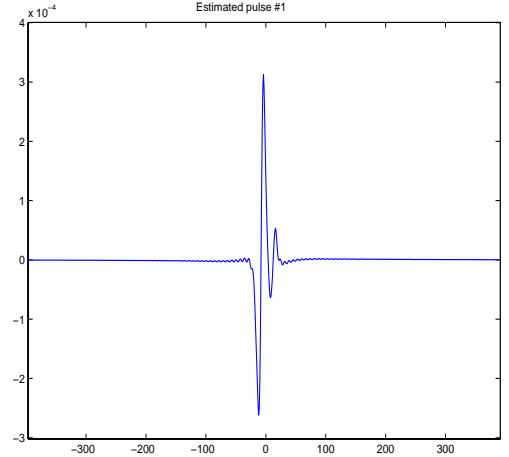

(c)

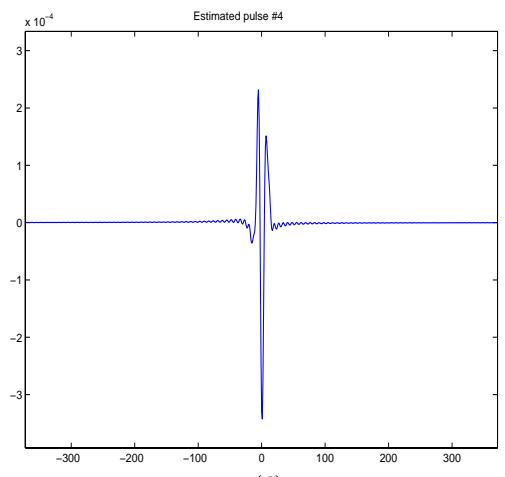

(f)

Fig. 3. Channel Estimation Performance (a) Received multipath signal (with 4 dominant components) made up of pulses having different pulse shapes. (b) Received noisy signal (blue) and the noiseless pulses (red). (c) Estimated shape of the first pulse. (d) Estimated shape of the second pulse. (e) Estimated shape of the third pulse. (f) Estimated shape of the fourth pulse. The received signal is sampled at one fourth the Nyquist rate. We used a polynomial of order $R=20$ to approximate the DFT coefficients of the received signal.

in frequency, and exclude the DFT coefficients that correspond to frequency bands of interfering signals. The results are shown for the case when SIR $=-20 \mathrm{~dB}$, which clearly indicate robustness of the method to strong narrowband interference.

\section{B. Channel Estimation Performance}

We next consider the case of the channel model given by (5). Specifically, we assume that a coded sequence of first-derivative Gaussian impulses is periodically transmitted, while a received (single) UWB signal is made up of multiple pulses having different shapes. We considered the case when there are 4 dominant closely spaced components, as illustrated in Fig. 3(a). The received noiseless and noisy UWB signals for SNR $=0 \mathrm{~dB}$ are shown in Fig. 3(b). The received signal is sampled uniformly at one fourth the Nyquist rate and the samples are averaged over 30 symbols. We first estimated the time delays of the dominant components by finding the roots of the annihilating filter. As already pointed out in Section III, the signal poles (and thus the unknown time delays) can be estimated by choosing $L=4$ zeros closest to the unit circle. Once the time delays of the pulses have been estimated, the corresponding pulse shapes are obtained by polynomial approximation of the DFT coefficients. In this case, we used a polynomial of degree $R=20$, which clearly yields a very good approximation of the received waveforms.
Effects of the sampling rate $N_{s}$ and the degree of the polynomial $R$ on the estimated pulse shape are illustrated in Figure 4. The sampling rate is varied between $N_{s}=$ $0.1 N_{n}$ and $N_{s}=0.25 N_{n}$, while the polynomial degree is chosen between $R=10$ and $R=20$. In general, by increasing the sampling rate and fitting the DFT coefficients with a polynomial of a larger degree, we obtain better estimates. For example, very good approximation of the pulse shape can already be obtained by sampling the signal at one fifth the Nyquist rate and using a polynomial of order $R=20$, as illustrated in Figure 4(e). It is also interesting to note that as the value of $R$ decreases, better performance can be achieved with lower sampling rates, which can be seen in Figures 4(e)-(f).

\section{Conclusions}

We presented a method for subspace channel estimation in ultra-wideband systems, which takes advantage of our recent sampling results for certain classes of parametric non-bandlimited signals. Our approach uses wellknown spectral estimation techniques and allows for highresolution channel estimation from the signal subspace. We summarize the appealing features of our framework:

- Allows for high-resolution channel estimation using subNyquist uniform sampling.

- Reduced sampling rate leads to reduced computational and power requirements. 


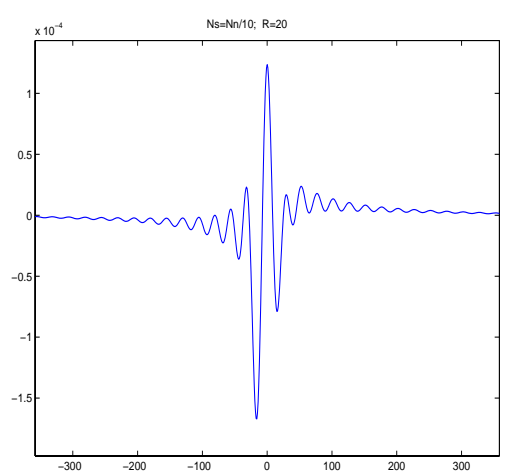

(a)

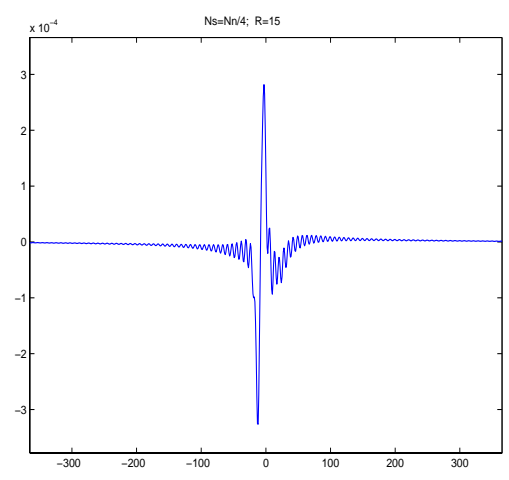

(d)

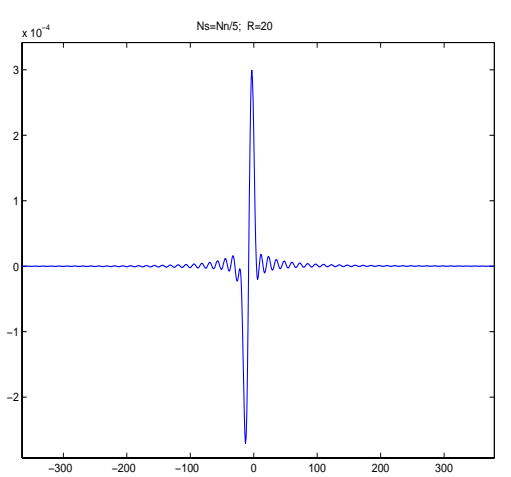

(b)

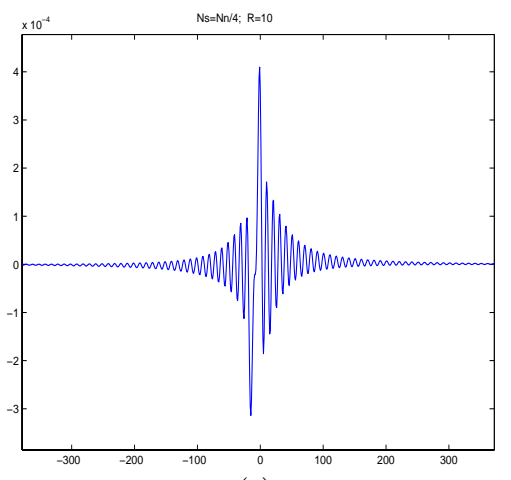

(e)

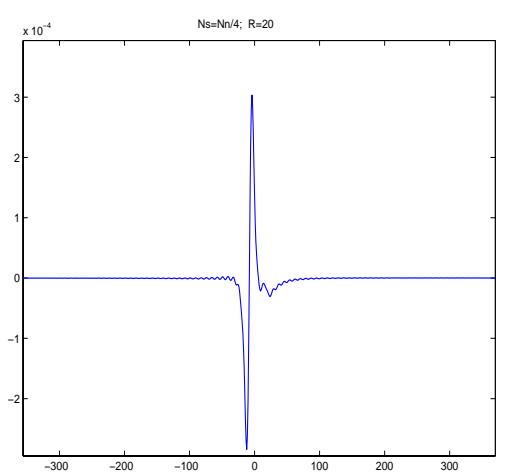

(c)

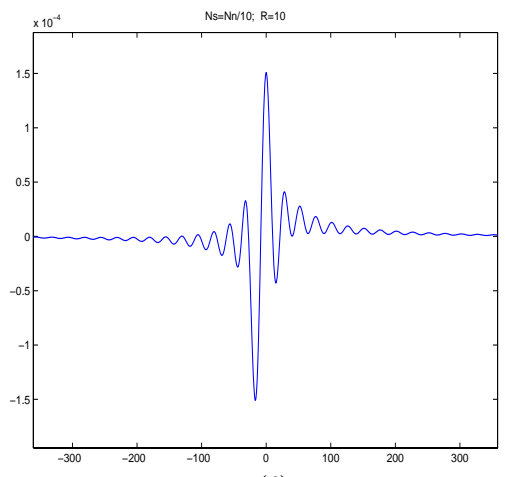

(f)

Fig. 4. Pulse shape estimation Estimated pulse shape (third pulse only) for different sampling rates $N_{s}$ as well as different values of the polynomial degree $R$. (a) $N_{s}=0.1 N_{n}, R=20$ (b) $N_{s}=0.2 N_{n}, R=20$ (c) $N_{s}=0.25 N_{n}, R=20$ (d) $N_{s}=0.25 N_{n}, R=15$ (e) $N_{s}=0.25 N_{n}, R=10$ (f) $N_{s}=0.1 N_{n}, R=10$.

- Uses fast algorithms and structured linear systems.

- Frequency bands used for estimation and sampling rates can be adapted to channel conditions.

Additionally, our framework allows for identification of more realistic channel models without resorting to complex algorithms. It is particularly suitable in applications such as estimation of wideband channels, precise position location or ranging. It can also be used in other wideband systems, such as CDMA, primarily for timing synchronization and localization purposes.

\section{REFERENCES}

[1] R. J. Cramer, R. A. Scholtz and M. Z. Win, "Evaluation of an Ultra-Wideband Propagation Channel", IEEE Trans. on Antennas and Propagation, Vol. 50, No. 5, pp 561-570, May 2002.

[2] M. Z. Win, R. A. Scholtz, "Impulse Radio: How it works", IEEE Commun. Letters, vol. 2, pp. 36-38, February 1998.

[3] M. Z. Win, R. A. Scholtz, "On the robustness of ultra-wide bandwidth signals in dense multipath environments", IEEE Commun. Letters, vol. 2, pp. 51-53, February 1998.

[4] M. Z. Win, R. A. Scholtz, "Characterization of ultra-wide bandwidth wireless indoor communication channel: A communication theoretic view", IEEE J. Select. Areas Commun., vol. 20, pp. 1613-1627, December 2002.

[5] D. Cassioli, M. Z. Win, A. F. Molisch, "The ultra-wide bandwidth indoor channel: From statistical model to simulations", IEEE J. Select. Areas Commun., vol. 20, pp. 1247-1257, December 2002

[6] I. O' Donnell, M. Chen, S. Wang and R. Brodersen," An Integrated, Low-Power, Ultra-Wideband Transceiver Architecture for Low-Rate Indoor Wireless System", IEEE CAS Workshop on Wireless Communications and Networking, September 2002.
[7] R. Fleming, C. Kushner, G. Roberts and U. Nandiwada, "Rapid Acquisition for Ultra-Wideband Localizers", in Proc. IEEE Conf. on UWB Systems and Technologies, May 2002.

[8] E. Homier and R. Scholtz, "Rapid Acquisition of UWB Signals in a Dense Multipath Channel," in Proc. IEEE Conf. on UWB Systems and Technologies, May 2002.

[9] J. Y. Lee and R. Scholtz, "Ranging in a dense multipath environment using an UWB radio link," IEEE Journal on Selected Areas in Communications, Vol. 20, No. 9, pp. 1677-1683, December 2002.

[10] M. Vetterli, P. Marziliano and T. Blu, "Sampling signals with finite rate of innovation," IEEE Transactions on Signal Processing, vol. 50. No. 6, pp. 1417-1428, June 2002.

[11] J. Kusuma, A. Ridolfi and M. Vetterli, "Sampling of communication systems with bandwidth expansion," in Proc. of ICC 2002, Vol. 3, pp. 1601-1605, May 2002.

[12] I. Maravic, M. Vetterli and K. Ramchandran, "High-Resolution acquisition methods for wideband communication systems," In Proc. of ICASSP, April 2003.

[13] J. Kusuma, I. Maravic and M. Vetterli, "Sampling with finite innovation rate: channel and timing estimation in UWB and GPS," In Proc. of ICC, May 2003.

[14] I. Maravic and M. Vetterli, "Low-complexity subspace methods for channel estimation and synchronization in ultra-wideband systems", In Proc. of IWUWB, June 2003.

[15] I. Maravic, M. Vetterli and K. Ramchandran, "High-resolution synchronization and channel estimation with sub-Nyquist sampling and application to ultra-wideband systems", LCAV Technical report and submitted to IEEE Trans. on Signal Processing.

[16] P. Stoica and R. Moses, Introduction to Spectral Analysis, Prentice Hall, 2000.

[17] Y. Hua and T. Sarkar, "Matrix pencil method for estimating parameters of exponentially damped/undamped sinusoids in noise", IEEE Transactions on Acoustics, Speech and Signal Processing, Vol. 38, No. 5, pp. 814-824, May 1990.

[18] R. Roy and T. Kailath, "ESPRIT estimation of signal parameters via rotational invariance techniques," IEEE Trans. on 
Acoustics, Speech and Signal Processing, vol. 37, No.7, pp. 984995, July 1989.

[19] B. D. Rao and K. S. Arun, "Model based processing of signals: A state space approach", Proceedings of the IEEE, Vol. 80, No. 2, pp. 283-309, February 1992.

[20] S. E. Bensley and B. Aazhang, "Subspace-based channel estimation for code division multiple access communication systems", IEEE Transactions on Communications, Vol. 44, No. 8., p.p. 1009-1020, August 1996.

[21] A. Paulraj, B. Khalaj and T. Kailath, "2-D RAKE receivers for CDMA cellular systems", in Proc. IEEE GLOBECOM, Vol. 1, San Francisco, CA, Dec. 1994, pp. 400-404.

[22] Q. Spencer, M. Rice, B. Jeffs, M. Jensen, "A Statistical Model for the Angle-of-Arrival in Indoor Multipath Propagation", in Proc. IEEE Vehicular Technology Conference, pp. 1415-1419, 1997.

[23] M. Z. Win, G. Chrisikos, N. R. Sollenberger, "Performance of Rake reception in dense multipath channels: Implications of spreading bandwidth and selection diversity order", IEEE J. Select. Areas Commun., vol. 18, pp. 1516-1525, August 2002.

[24] P. Stoica and A. Nehorai, "MUSIC, Maximum Likelihood and Cramer-Rao Bound", IEEE Trans. on Acoustics, Speech and Signal Processing, vol. 37, No. 5, pp. 720-741, May 1989.

[25] L. R. Rabiner and B. Gold, "Theory and Application of Digital Signal Processing", Prentice-Hall, Englewood Cliffs, New Jersey, 1975 